\title{
Cystatin $\mathrm{C}$ as regulator of autophagy in brain of transgenic mice with model of Parkinson's disease
}

\author{
A.B. Shintyapina ${ }^{1}$, T.A. Korolenko ${ }^{2 *}$, A.B. Pupyshev², E.L. Zavjalov ${ }^{3}$, \\ T.G. Amstislavskaya ${ }^{2,4}$, M.A. Tikhonova ${ }^{2,4}$ \\ ${ }^{1}$ Institute of Molecular Biology and Biophysics of Federal Research Center of Fundamental \\ and Translational Medicine, Novosibirsk, Russia \\ ${ }^{2}$ Institute of Physiology and Basic Medicine, Novosibirsk, Russia \\ ${ }^{3}$ Institute of Cytology and Genetics SB RAS, Novosibirsk, Russia \\ ${ }^{4}$ Novosibirsk State University, Novosibirsk, Russia \\ *e-mail: t.a.korolenko@physiol.ru
}

Key words: Cystatin C, autophagy, transgenic model of Parkinson's disease

Motivation and Aim: Autophagy was shown to be suppressed in striatum of transgenic mice with model of Parkinson's disease [1]. Cystatin $\mathrm{C}$ is one of the potent regulators of autophagy [2]. Changes in the expression and secretion of Cystatin $\mathrm{C}$ in the brain have been shown in amyotrophic lateral sclerosis, Alzheimer's and Parkinson's diseases and in some animal models of neurodegeneration, demonstrating protective role of Cystatin $\mathrm{C}$ [3]. It was suggested that Cystatin $\mathrm{C}$ plays the primary role in amyloidogenesis and perspective as treatment of neurodegenerative diseases. Cystatin $\mathrm{C}$ colocalizes with amyloid beta-protein in brain in Alzhemer's disease. Controlled expression of a cystatin C-peptide was suggested as a new approach to therapy for Alzheimer's disease. In Parkinson's disease serum Cystatin C levels can predict disease severity and cognitive dysfunction, although the exact role of Cystatin $\mathrm{C}$ remains unclear. The aim: to evaluate expression of Cystatin $\mathrm{C}$ in transgenic mice with model of Parkinson' disease in early pathological state of disease (5 months) and evaluate results as related to mechanism of development of autophagy.

Methods and Algorithms: 5-month-old male mice ofB6.Cg-Tg(Prnp-SNCA*A53T)23Mkle/J) (further - B6.Cg-Tg) and control C57B1/6J strain were used. Total RNA was purified from mouse brain areas (striatum, amygdaloid complex, hypothalamus, hippocampus) using RNeasy Plus mini kit (Qiagen). qPCR was performed in a CFX96 Real-Time PCR Detection System (Bio-Rad, USA) using HS-qPCR Mix SYBR Green (2x) (Biolabmix, Russia), $200 \mathrm{nM}$ real-time PCR primers (Forward Primer $\left(5^{\prime} \rightarrow 3^{\prime}\right)$ AGGAGGCAGATGCCAATGAG; Reverse Primer $\left(5^{\prime} \rightarrow 3^{\prime}\right)$ GGGCTGGTCATGGAAAGGA), $5 \mu 1$ template $(1: 50$ diluted cDNA).

Results: Cystatin C (Cst3) gene expression analysis in striatum and, especially in amygdaloid complex, in mice with transgenic model of Parkinson's disease (5 months) revealed statistically significant ( $p=0.0168$ ) decrease vs control; there was a correlation between the Cst 3 expression and marker of autophagy LC32 level (immunohistochemistry). Cystatin C concentration in serum (ELISA) of transgenic mice was not changed $v s$ control.

Conclusion: The data obtained confirms that Cst3 expression in striatum correlates with autophagy. Cystatin $\mathrm{C}$ can play a protective role in multiple neurodegenerative disorders, including Parkinson's and Alzheimer's diseases.

Acknowledgements: Supported partially by grant No. 16-04-01423-a from the Russian Foundation for Basic Research (Russia). Unique scientific installation "Biological collection - Genetic biomodels of neuro-psychiatric disorders" (No. 493387) Scientific Research Institute of Physiology and Basic Medicine. The studies implemented using the equipment of the Center for Genetic Resources of Laboratory Animals at ICG SB RAS, supported by the Ministry of Education and Science of Russia (Unique identifier of the project RFMEFI62117X0015).

\section{References}

1. Pupyshev A.B., Korolenko T.A., Akopyan A.A., Amstislavskaya T.G., Tikhonova M.A. (2017) Neurosci. Lett. pii: S0304-3940(17)30974-6.

2. Zou J., Chen Z., Wei X., Chen Z., Fu Y., Yang X., Chen D., Wang R., Jenner P., Lu J.H., Li M., Zhang Z., Tang B., Jin K., Wang Q. (2017) Cell Death Disease. 8:e2854.

3. Kaur G., Levy E. (2012) Front. Mol. Neurosci. 5:79. 